\title{
Use of swabs for dry collection of self- samples to detect human papillomavirus among Malagasy women
}

Pierre Vassilakos ${ }^{1}$, Rosa Catarino ${ }^{2 *}$, Stephanie Bougel ${ }^{3}$, Maria Munoz ${ }^{1}$, Caroline Benski ${ }^{2,4}$, Ulrike Meyer-Hamme ${ }^{2}$, Jeromine Jinoro ${ }^{4}$, Josea Lea Heriniainasolo ${ }^{4}$ and Patrick Petignat ${ }^{2}$

\begin{abstract}
Background: Most women in developing countries have never attended cervical screening programmes and often little information exists on type-specific human papillomavirus (HPV) prevalence among these populations. Selfsampling for HPV testing (self-HPV) using a dry swab may be useful for establishing a screening program and evaluating HPV prevalence. Our aim was to evaluate self-HPV using a dry swab stored at room temperature.

Methods: This community-based study in Madagascar consisted of 449 women aged 30-65. Eligible women were provided a dry swab to perform self-HPV. HPV analysis was accomplished by two different real-time PCR tests using the same extracted DNA from the samples.

Results: Overall, 52 (11.6\%) specimens were invalid for HPV detection. The delay between sampling and laboratory processing of DNA extraction considerably increased invalid results. Overall HPV prevalence of 14 hrHPV types detected by the two PCR tests was found to be $38.2 \%(n=152)$. Distribution of 19 hrHPV and 9 low-risk HPV (IrHPV) types revealed most frequently 53 and 68 among hrHPV and HPV 54, HPV 70 and HPV 42 among IrHPV. Agreement between the two PCR methods for any of the 14 high-risk HPV (hrHPV) strains detected was $89.9 \%$ (kappa $=0.77,95 \% \mathrm{Cl}: 0.71-0.84)$. In 385 (85.7\%) samples the DNA load of ß-globin demonstrated a signal with medium or high level copies. Conversely, in 28 (60.9 \%) invalid samples the signal was undetectable. The HPV-DNA load signal was predominantly of intermediate level $(58.5 \%, n=218)$.
\end{abstract}

Conclusions: Self-HPV using a dry swab stored at room temperature could be a useful method for HPV screening and for conducting population-based surveys on HPV prevalence in resource-poor settings.

Keywords: Self-sampling, Dry swab, Human papillomavirus (HPV), HPV testing, HPV prevalence, Cervical cancer

\section{Background}

The high sensitivity of clinically validated HPV tests together with the limitations associated with a Pap test [1], led to the recommendation of HPV testing as a replacement of cytology for primary screening in the past decade [2]. Besides these issues, logistic difficulties to introduce cytology-based screening programs in lowand medium-income countries (LMIC) led to the validation of high-risk HPV (hrHPV) testing as an alternative

\footnotetext{
* Correspondence: rosapintocatarino@gmail.com

${ }^{2}$ Division of Gynaecology, Department of Gynaecology and Obstetrics, Geneva University Hospitals, Boulevard de la Cluse 30, 1206 Geneva, Switzerland

Full list of author information is available at the end of the article
}

for primary screening by the World Health Organization [3]. However, a screening program is only successful when most of the eligible women participate. In LMIC, many women are unable to visit healthcare facilities, and cervical cancer $(\mathrm{CC})$ screening programs requiring speculum examination are difficult to implement because of logistical issues. A promising alternative for these women is screening by means of self-HPV testing (self-HPV) [4].

Several studies have shown that Self-HPV is acceptable to women [5-7] and compares favourably to cliniciancollected specimens $[5,8-10]$. A recent meta-analysis showed that when PCR-based HPV tests were used, the clinical performance of self-HPV was similar to 
clinician-collected samples, irrespective of the type of device used [11].

Self-HPV implementation in LMIC requires a careful analysis of logistics. The choice of HPV test and collection device is of paramount importance. Optimization of transport and storage still remains a challenging issue. Most of the collection devices are stored in specimen transport medium or liquid-based cytology media, which preserve HPV-DNA at room temperature but are expensive and unavailable in a resource-poor context. Additionally, they are toxic and flammable and spillage and leakage can happen during collection and transit.

Compared with liquid media, dry swabs have the advantage of being less expensive and not requiring a special media, so the sample can be safely transported at ambient temperature. Studies demonstrated a good agreement (70-92\%) for HPV detection between dry and wet swabs [12-15].

The goal of this study was to determine, within field conditions of $\mathrm{CC}$ screening in Madagascar, the validity of HPV detection in self-obtained samples using a dry swab stored at room temperature. Additionally, we analysed HPV prevalence and genotype distribution among the screened population.

\section{Methods}

\section{Study setting}

The study was conducted by the University of Geneva in collaboration with Madagascar's Health and Family Planning Ministry and the Saint-Damien Healthcare Centre in Ambanja, a mainly rural area with 125,056 inhabitants.

All participants signed an informed consent form before inclusion. The study was conducted from February to March 2015 in the Saint-Damien Healthcare Centre. Ethical approval of the study was obtained from the Malgasch National Commission for the Ethics of Science and Technology, as well as from the Ethical Cantonal Board of Geneva, Switzerland (CER: 14-071).

\section{Study participants and procedures}

We recruited 449 women aged between 30 and 65 years. Exclusion criteria were former conisation or hysterectomy and pregnancy beyond 20 weeks.

Participants received educational intervention on cancer and HPV infection. It was followed by information on how to use the self-HPV, written in the local dialect language. At the same time the participants received a sterile, cotton-tipped swab in a dry, labelled tube. SelfHPV was always performed without supervision. Then participants were invited to answer a questionnaire regarding socio-demographic information. HPV tests were analysed in Switzerland. HPV test analysis was done in a minimum time of 15 days after the sampling.
HPV-negative women were informed that they were not at risk for $\mathrm{CC}$ and that they would not require a test within the next 5 years. HrHPV-positive women were invited for colposcopic examination. A biopsy on acetowhite lesions coupled with endocervical curettage was performed, or a biopsy at the 6 o'clock position and endocervical curettage if colposcopy was normal. Treatment and follow-up was proposed according to the histological diagnosis. In case of an invalid result, women were asked to repeat their self-sampling. Histology was also analysed in Switzerland.

\section{Laboratory methods}

Upon arrival to the laboratory, the swabs were suspended in $4.3 \mathrm{~mL}$ of $\operatorname{cobas}^{\circ} \mathrm{PCR}$ media and were pulse vortexed for $3 \times 10 \mathrm{~s}$. A volume of $400 \mu \mathrm{L}$ of each sample was used for DNA extraction and the rest of the sample was stored at $4{ }^{\circ} \mathrm{C}$. DNA extraction was carried out using the cobas $^{\circ}$ HPV Test (Roche Molecular Systems). Nucleic extracts were then stored at $-20{ }^{\circ} \mathrm{C}$.

HPV analysis was accomplished by two different realtime PCR tests: the cobas ${ }^{\oplus}$ HPV Test (cobas) and the Anyplex $^{\text {Ti }}$ II HPV28 (H28) test (Seegene, Seoul, South Korea), using the same extracted DNA from samples.

Amplification and detection were first carried out with cobas (at Biopath Lab, Lausanne), which detects 12 pooled hrHPV genotypes (HPV 31, 33, 35, 39, 45, 51, 52, $56,58,59,66$, and 68 ) and concurrently provides separate results for HPV-16 and HPV-18. The detection is based on amplification of the L1 gene and TaqMan probes [16]. The human reference gene $\beta$-globin s also detected.

Amplification and detection using the stored DNA extracts were then performed with the $\mathrm{H} 28$ test using the CFX96 ${ }^{\text {Tu }}$ real-time thermocycler (at Buhlmann Laboratories AG Schönenbuch, Switzerland). Data recording and interpretation were automated. Details of the procedure and evaluation were described by Estrade et al. [17], Kim et al. [18] and Kwon et al. [19].

$\mathrm{H} 28$ is a semi-quantitative real-time multiplex PCR assay for screening and HPV genotyping. This test uses Dual Priming Oligonucleotides $\left(\mathrm{DPO}^{\mathrm{m}}\right)$ and Tagging Oligonucleotide Cleavage and Extension $\left(\mathrm{TOCE}^{\mathrm{nm}}\right)$ technologies and allows to simultaneously detect 19 high-risk HPVs (including types 16, 18, 26, 31, 33, 35, 39, 45, 51, 52, 53, 56, 58, 59, 66, 68, 69, 73 and 82) and 9 low-risk HPVs (including types 6, 11, 40, 42, 43, 44, 54, 61 and 70). ß-globin is also detected for internal control of assay validity.

Knowledge of the step at which the melting curve becomes positive allows for semi-quantification of the DNA load of $ß$-globin gene and HPV genomes, from low (+; positive after 40 PCR cycles, $<10^{2}$ copies/reaction), to intermediate $\left(++\right.$; positive within 31 to 39 PCR cycles, $\geq 10^{2}$ and $<10^{5}$ copies/reaction), to high (+++; positive before 31 PCR cycles, $\geq 10^{5}$ copies/reaction) (17). 


\section{Data analysis and statistics}

Data were analysed with STATA 13 software package (StataCorp, Texas, USA).

Inter-rater agreement statistics and kappa coefficient with $95 \%$ confidence intervals, percent total agreement and percent positive agreement were calculated for the paired results obtained by the cobas and H28 tests. The calculation was restricted to $14 \mathrm{hrHPV}$.

The trend of association between HPV results and DNA load was evaluated with the chi-square test. We also evaluated the effect of transport time on sample degradation by applying a Kaplan-Meier failure estimate.

The HPV prevalence was calculated from the number of positive cases divided from the number of tested specimens by both PCR methods. HPV positivity was distributed by age-group.

HPV type distribution in single and multiple infections of 19 hrHPVs and 9 lrHPVs was explored using the $\mathrm{H} 28$ test.

\section{Results}

\section{Sample characteristics}

The study included 449 self-obtained swab specimens from women whose median age was 43 (IQR: 36-51). Overall, 52 (11.6\%) specimens were invalid for HPV detection, 44 (9.8 \%) were invalid for cobas, 46 (10.0\%) were invalid for $\mathrm{H} 28$ and 38 (73.1 \%) were concordantly classified as invalid: 6 were invalid by $\mathrm{H} 28$ but negative by cobas, 5 were invalid by cobas but negative by H28, 1 was invalid by cobas but positive by $\mathrm{H} 28$ and 2 were invalid by $\mathrm{H} 28$ but positive by cobas (data not shown).

The overall prevalence for the 14 hrHPV types detected by one of the two methods was $38.2 \%(152 / 397)$; $19.7 \%(30 / 152)$ of samples were positive for HPV-16/18, while $88.2 \%(134 / 152)$ were positive for the pooled 12 hrHPV types. The overall prevalence for $19 \mathrm{hrHPV}$ and 9 lrHPV was $51.8 \%$ (209/403); the prevalence for hrHPV only was $38.6 \%$ (156/403).

Overall, $96 \mathrm{HPV}$-positive patients came to the colposcopy clinic. There were $6(6.3 \%)$ cervical intraepithelial neoplasia grade 1 (CIN1), 4 (4.2\%) CIN grade 2 (CIN2), 4 (4.2\%) CIN grade 3 (CIN3) and 2 (2.1\%) squamous cell carcinomas. The overall CIN2 or more severe (CIN2+) prevalence was $10.4 \%$.

\section{Agreement between cobas and H28 for HPV-DNA detection by type categories}

The agreement was $89.9 \%$ for any HPV type, $97.5 \%$ for HPV-16/18 and $90.7 \%$ for the category with HPV pooled types. The proportion of positive agreement was 84.8, 80.0 and $84.0 \%$ for any hrHPV, HPV-16/18 and pooled HPVs, respectively (Table 1 ).
$\mathrm{H} 28$ test results and semi-quantification of ß-globin and HPV-DNA load, stratified on delay between sampling and laboratory processing of DNA extraction ( 2 to $\geq \mathbf{4}$ weeks) Overall, in $85.7 \%$ of the samples the DNA load of $ß$-globin demonstrated a signal with medium- or high-level copies. In $60.9 \%$ of invalid samples the signal was undetectable. For $7 \mathrm{HPV}$-positive cases the ß-globin was undetectable (Table 2). There were no significant differences in the number of invalid samples nor in DNA load of $\beta$ globin between pre- ( $\leq 50$ years $)$ and postmenopausal ( $>50$ years) women ( $p=0.834$ and $p=0.290$, respectively).

The delay between sampling and laboratory processing increased invalid results from $5.7 \%$ after 2 weeks to $9.4 \%$ after 3 weeks and $12.6 \%$ after $\geq 4$ weeks $(p=0.177)$. The mean delay between sampling and laboratory processing was $22.7 \pm 0.35$ days for valid samples and 24.3 days for invalid samples ( $p=0.129)$. In Fig. 1, a Kaplan-Meier failure estimate showing a clear increase in the proportion of invalid tests with time.

Analysis of the relationship between HPV positive test results and DNA load (Table 3) showed that the strength of the load signal was predominantly intermediate (58.5\%). The delay between sampling and DNA extraction did not interfere on the strength of the signal $(p=0.206)$. The strength of the HPV-DNA load was not related to the strength of the $\beta$-globin DNA load $(p=0.154)$. There were no significant differences in HPV-DNA load between preand postmenopausal women $(p=0.806)$ (data not shown).

\section{Age-specific HPV prevalence according to cobas and $\mathrm{H} 28$ real-time PCR tests}

The overall prevalence for $14 \mathrm{hrHPV}$ types was found to be $32.1 \%$ with cobas and $29.5 \%$ with H28. Stratification by age demonstrates a decrease from the age group of 3035 years to the age group $36-45$ years. Then a rebound is observed in the age group of 46-55 years, followed by a decrease in the age group of 56-65 years (Fig. 2).

\section{Type-specific prevalence in mono- and multi-infections according to $\mathrm{H} 28$ real-time PCR test (Table 4)}

Overall, $31.6 \%$ of the HPV infections were single whereas $68.4 \%$ were multiple. Sixty-one percent of women's infections revealed hrHPV, particularly HPV-53 and 68, followed by HPV-73, HPV-52, HPV-35, HPV-16, HPV-33, HPV-31 and HPV-18 in decreasing order. Infections with low risk types disclosed particularly HPV-54, HPV-70 and HPV-42, followed by HPV-44, HPV-40 and HPV-6 in decreasing order. There were no significant differences in the distribution of mono-infections vs. multi-infections for hr- and lrHPVs $(p=0.300$ and $p=0.202$, respectively).

\section{Discussion}

In Madagascar, besides the lack of effective CC screening, population-based surveys on HPV prevalence are 
Table 1 Agreement between cobas and H28 for HPV-DNA detection by type categories

\begin{tabular}{llll}
\hline Results cobas/H28 & HPV distribution by type categories & & \\
\cline { 2 - 4 }$N(\%)$ & Any hrHPV & HPV16/18 & Pool: 31, 33, 35, 39, 45, \\
& & & $51,52,56,58,59,66,68$ \\
\hline Pos/Pos & 112 & 20 & 97 \\
Pos/Neg & 16 & 5 & 15 \\
Neg/Pos & 24 & 5 & 22 \\
Neg/Neg & 245 & 367 & 263 \\
Total & 397 & 397 & 397 \\
Kappa (95 \% Cl) & $0.77(0.71-0.84)$ & $0.79(0.66-0.92)$ & $0.77(0.71-0.84)$ \\
\% Total Agreement & 89.9 & 97.5 & 90.7 \\
\% Positive Agreement ${ }^{c}$ & 84.8 & 80.0 & 84.0 \\
P value & $<0.001$ & $<0.001$ & $<0.001$
\end{tabular}

Abbreviations: $H 28$ Seegene Anyplex ${ }^{\mathrm{TM}}$ II HPV28, Pos Positive, Neg Negative, $N$ number

aAny high-risk HPV: HPV 16 and/or HPV18 and/or HPV 31, 33, 35, 39, 45, 51, 52, 56, 58, 59, 66, 68

bHPV16/18: HPV16 and or HPV18

'The proportion of positive agreement between paired cobas and $\mathrm{H} 28$ samples was calculated using $2 \mathrm{a} /(\mathrm{f} 1+\mathrm{g} 1)$, where $\mathrm{a}$ is the number of samples that were positive for HPV in both tests, $\mathrm{f} 1$ is the number of samples that were positive for cobas, and $\mathrm{g} 1$ is the number of samples that were positive for $\mathrm{H} 28$

Table 2 H28 HPV results and semi-quantification of ß-globin DNA load, stratified on delay between sampling and laboratory processing of DNA extraction (2 to $\geq 4$ weeks)

\begin{tabular}{|c|c|c|c|c|c|c|}
\hline \multirow[t]{3}{*}{ HPV results } & \multicolumn{5}{|c|}{ B-globin DNA load } & \multirow[t]{3}{*}{$p$ value } \\
\hline & Undetectable & + & ++ & +++ & Total & \\
\hline & $N(\%)$ & $N(\%)$ & $N(\%)$ & $N(\%)$ & $N(\%)$ & \\
\hline \multicolumn{7}{|l|}{2 weeks } \\
\hline Positive & $1(2.3)$ & $1(2.3)$ & $32(74.4)$ & $9(20.9)$ & $43(100.0)$ & $<0.001$ \\
\hline High-risk ${ }^{b}$ & 0 & $1(3.3)$ & $23(76.7)$ & $6(20.0)$ & $30(100.0)$ & \\
\hline Low-risk ${ }^{c}$ & $1(4.6)$ & 0 & $17(77.3)$ & $4(18.2)$ & $22(100.0)$ & \\
\hline Negative & 0 & 0 & $26(65.0)$ & $14(35.0)$ & $40(100.0)$ & \\
\hline Invalid & $3(60.0)$ & $2(40.0)$ & 0 & 0 & $5(100.0)$ & \\
\hline \multicolumn{7}{|l|}{3 weeks } \\
\hline Positive & $2(3.5)$ & $4(7.0)$ & $38(66.7)$ & $13(22.8)$ & $57(100.0)$ & $<0.001$ \\
\hline High-risk $^{b}$ & $1(2.3)$ & $4(9.1)$ & $30(68.2)$ & $9(20.5)$ & $44(100.0)$ & \\
\hline Low-risk $^{c}$ & $1(3.9)$ & $2(7.7)$ & $18(69.2)$ & $5(19.2)$ & $26(100.0)$ & \\
\hline Negative & 0 & 0 & $50(72.5)$ & $19(27.5)$ & $69(100.0)$ & \\
\hline Invalid & $5(38.5)$ & $8(61.5)$ & 0 & 0 & $13(100.0)$ & \\
\hline$\geq 4$ weeks & & & & & & $<0.001$ \\
\hline Positive & $4(3.7)$ & $6(5.5)$ & $79(72.5)$ & $20(18.4)$ & $109(100.0)$ & \\
\hline High-risk ${ }^{b}$ & $2(2.4)$ & $5(6.1)$ & $57(69.5)$ & $18(22.0)$ & $82(100.0)$ & \\
\hline Low-risk ${ }^{c}$ & $3(4.6)$ & $3(4.6)$ & $51(78.5)$ & $8(12.3)$ & $65(100.0)$ & \\
\hline Negative & 0 & 0 & $62(72.9)$ & $23(27.1)$ & $85(100.0)$ & \\
\hline Invalid & $20(71.4)$ & $8(28.6)$ & 0 & 0 & $28(100.0)$ & \\
\hline TOTAL & $35(7.8)$ & $29(6.5)$ & $287(63.9)$ & $98(21.8)$ & 449 (100.0) & \\
\hline
\end{tabular}

\section{Abbreviations: $N$ number}

a Undetectable, absence of signal; + (low), positive $>40$ PCR cycles, $<10^{2}$ copies/reaction; ++ (medium) positive within 31 to 39 PCR cycles, $\geq 10^{2}$ and $<10^{5}$ copies/ reaction; +++ (high) positive before $31 \mathrm{PCR}$ cycles, $\geq 10^{5}$ copies/reaction

${ }^{b}$ Positivity for a high-risk HPV type $(16,18,26,31,33,35,39,45,51,52,53,56,58,59,66,68,69,73$ and 82$)$

'Positivity for a low-risk type (6, 11, 40, 42, 43, 44, 54, 61 and 70)

NB For multiple infections positive results were pooled and a single result was generated 


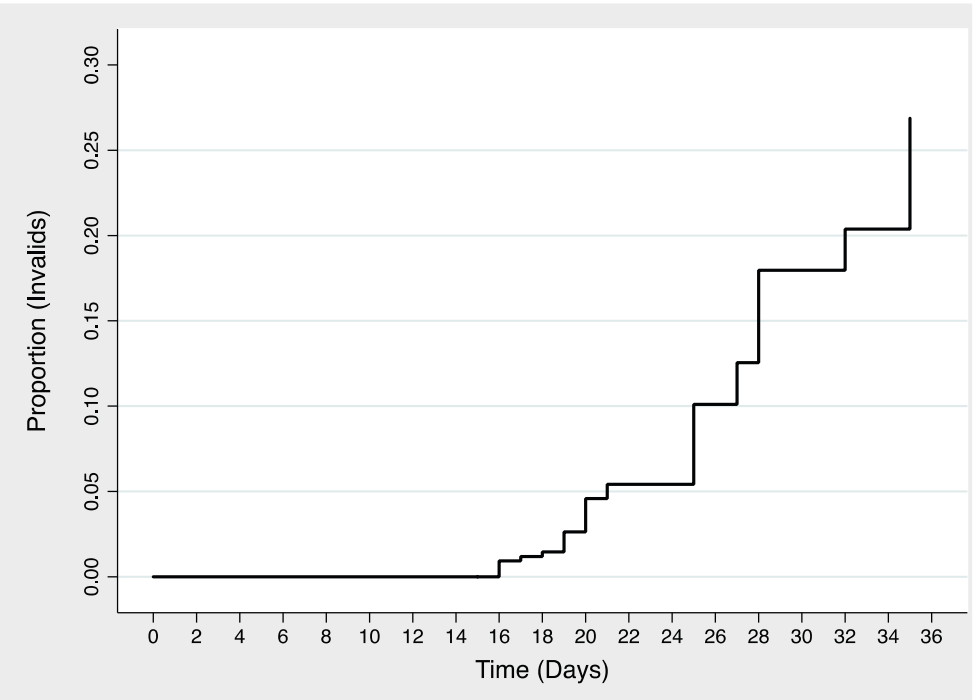

Fig. 1 Kaplan-Meier failure estimate showing the proportion of H28 invalid HPV tests over the days. HPV test analysis was done in a minimum time of 15 days following the sampling

not yet available [20]. In view of these considerations, this study was designed to assess the validity of self-HPV using dry swabs.

Our results indicate that dry swabs provided sufficient amounts of biological material and stable DNA for

Table 3 H28 HPV positive results and semi-quantification of HPV-DNA load, stratified on delay between sampling and laboratory processing of DNA extraction ( 2 to $\geq 4$ weeks)

\begin{tabular}{|c|c|c|c|c|c|}
\hline \multirow{3}{*}{$\begin{array}{l}\text { HPV } \\
\text { positive } \\
\text { results }\end{array}$} & \multicolumn{4}{|c|}{ HPV-DNA load ${ }^{a}$} & \multirow[t]{3}{*}{$p$ value } \\
\hline & + & ++ & +++ & Total & \\
\hline & $N(\%)$ & $N(\%)$ & $N(\%)$ & $N(\%)$ & \\
\hline \multicolumn{6}{|l|}{2 weeks } \\
\hline High-risk $^{\mathrm{b}}$ & $20(44.4)$ & $25(55.7)$ & 0 & $45(100.0)$ & 0.009 \\
\hline Low-risk ${ }^{c}$ & $9(34.6)$ & $12(46.2)$ & $5(19.2)$ & $26(100.0)$ & 0.009 \\
\hline \multicolumn{6}{|l|}{3 weeks } \\
\hline High-risk ${ }^{b}$ & $22(37.9)$ & $30(51.7)$ & $6(10.3)$ & $58(100.0)$ & 0.919 \\
\hline Low-risk ${ }^{c}$ & $11(34.4)$ & $18(56.3)$ & $3(9.4)$ & $32(100.0)$ & 0.919 \\
\hline \multicolumn{6}{|l|}{$\geq 4$ weeks } \\
\hline High-risk ${ }^{b}$ & $38(30.4)$ & $75(60.0)$ & $12(9.6)$ & $125(100.0)$ & 0.409 \\
\hline Low-risk ${ }^{c}$ & $20(21.0)$ & $58(66.7)$ & $9(10.3)$ & $87(100.0)$ & 0.491 \\
\hline TOTAL & $120(32.2)$ & $218(58.5)$ & $35(9.4)$ & $373(100.0)$ & \\
\hline
\end{tabular}

Abbreviations: $N$ number

a + (low), positive $>40 \mathrm{PCR}$ cycles, $<10^{2}$ copies/reaction; ++ (medium) positive within 31 to 39 PCR cycles, $\geq 10^{2}$ and $<10^{5}$ copies/reaction; +++ (high) positive before 31 PCR cycles, $\geq 10^{5}$ copies/reaction

${ }^{\text {b} P o s i t i v i t y ~ f o r ~ a ~ h i g h-r i s k ~ H P V ~ t y p e ~}(16,18,26,31,33,35,39,45,51,52,53,56$, $58,59,66,68,69,73$ and 82 )

'Positivity for a low-risk type $(6,11,40,42,43,44,54,61$ and 70$)$

NB: For multiple infections each single positive result was counted for high- and low-risk types
hrHPV detection by two PCR-based tests (cobas and H28). Agreement between the two real-time PCR assays for the detection of any HPV among the 14 hrHPV types was $89.9 \%$ (kappa $=0.77)$, despite different technologies and cut-off algorithms. A similarly-strong agreement was observed after the hierarchical categorization of hrHPV types into two groups, according to cancer risk (HPV-16/18 and pool of others).

We investigated the quality of the samples by analysing the relationship between HPV test results and the DNA load. The human reference gene ß-globin, a marker for cellularity, and the HPV-DNA for $19 \mathrm{hrHPV}$ and $9 \mathrm{lrHPV}$ were semi-quantified. Our findings are in line with other studies indicating that the quality of a dry specimen is sufficient for HPV detection [12] but the delay between sampling and HPV detection may interfere in the successful amplification of the reference gene for internal control, generating invalid results [21, 22]. The failure to detect DNA load over time is probably because of genomic DNA degradation in some samples [21]. Baay et al. [22] compared ß-globin concentrations and purity in vaginal samples self-collected by college students on-site to samples that were collected at home and mailed to the study laboratory with a delay ranging from 1 to 23 days (mean 4 days). They observed that DNA yield decreased with longer transport time; however, this had only a minimal effect on PCR amplification. Lin et al. [23], using a referral population, evaluated the stability of samples collected with dry swabs and tested with the cobas $4800 \mathrm{HPV}$ test. They found that sample stored at an ambient, uncontrolled temperature can last up to one month 


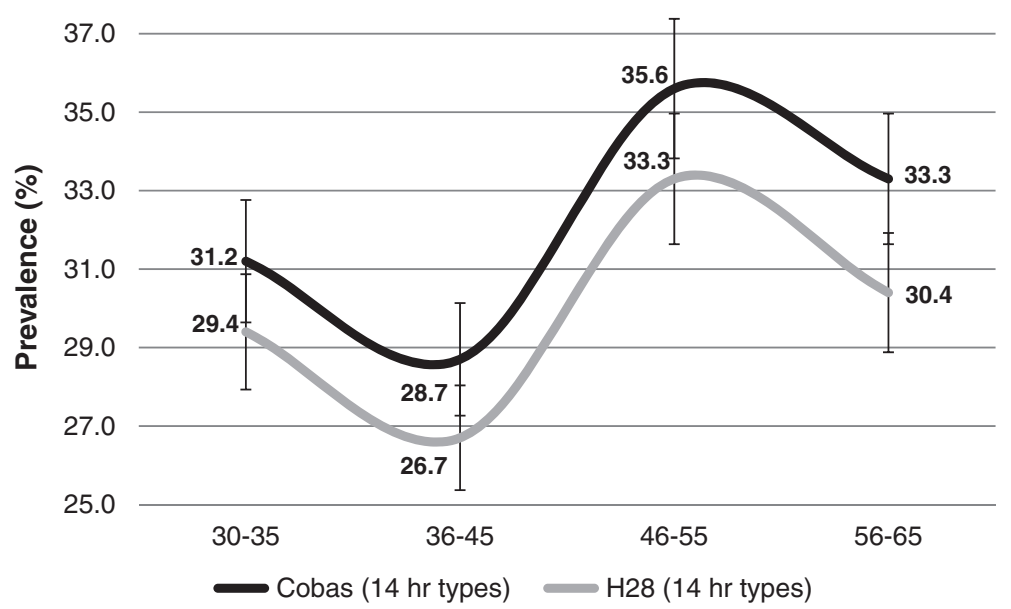

Fig. 2 Age-specific HPV prevalence (\%) according to cobas and H28 real-time PCR tests. Abbreviations: H28= Seegene Anyplex II HPV28; hr= high-risk; NB: For multiple infections, results were pooled and a single positive result was generated

without loss of sensitivity and specificity for detecting high-grade cervical intraepithelial lesion or cancer. Our findings confirm that stability of DNA in the sample collected with a dry swab is maintained, however, specimens processed after 2 weeks were more likely to be invalid for analysis. Concerning the strength of the HPV-DNA load, no detectable impact of the transport time was found on the signal strength $(p=0.206)$ and the strength of HPVDNA load was not related to the strength of the $B$-globin DNA load $(p=0.154)$. Interestingly, in 7 samples with undetectable B-globin, PCR analysis with $\mathrm{H} 28$ revealed positive HPV-DNA. This finding may corroborate the hypothesis that HPV-DNA present predominantly as episomal copies in dry samples is less affected from degradation [21].

Our data show that menopausal status does not affect the quality of samples. Nevertheless, it should be noted that besides DNA degradation because of the transportation time, undetectable DNA may also be the result of an inadequate sampling or the presence of PCR inhibitors [24].

This study provides for the first time information on age-specific HPV prevalence in screened women in Madagascar. Cobas and H28 were in agreement for the overall prevalence rates found for $14 \mathrm{hrHPV}$ types. The pattern of HPV prevalence for the restricted age-range of our study appeared similar to some surveyed African countries, but different to others [25]. The observed peak among women aged 45 years and older may be explained by newly acquired infections or by reactivation of latent HPV infections [26].

The high HPV prevalence found among the screened Malagasy women is consistent with published metaanalyses for Eastern Africa. Systematic reviews reported a HPV prevalence of $33.6 \%$ [25] and $42.2 \%$ [27] in women with normal cytology. The comparison of our data on HPV type distribution with other African countries $[27,28]$, indicates that the epidemiology of HPV infection is different in the study area. Differences in age pattern of HPV prevalence and type distribution in countries and regions may be related to different sexual habits and migrations of people [29].

In this study we used simple cotton swab for sample collection. Flocked swabs were found to have higher sensitivity to detect HPV infection compared to Dacron swabs [30], which was explained by its higher capacity of adhesion leading to a better proportion of DNA. However, in more recent studies [31, 32], no difference was found between the two swabs types for DNA retrieval.

We do recognize that the approach for HPV testing and subsequent follow-up applied in this study is unrealistic for a routine CC screening program in Madagascar. Besides invalid samples, a long delay between screening and reporting the results may lead to increased dropout of HPV-positive women who need to be recalled for management. A solution is the use of emergent rapid PCR-based methods for detecting HPV-DNA, with minimal requirements for laboratory equipment, enabling primary screening and treatment in a single visit. Another limitation in our analysis is the lack of histopathological results for all tested women, which would be useful as gold-standard for both PCR methods.

Because estimates of HPV prevalence and typespecific distribution in Madagascar are necessary for orienting CC prevention and monitoring the impact of vaccination, our study needs to be completed with estimates of a wider age range (15-60 years). 
Table 4 Type-specific prevalence in mono and multi-infections according to the $\mathrm{H} 28$ real-time PCR test

\begin{tabular}{|c|c|c|c|c|}
\hline \multirow{2}{*}{$\begin{array}{l}\text { HPV type } \\
\text { distribution }\end{array}$} & Mono-infections & Multi-infections & Total & \multirow[t]{2}{*}{$p$ value * } \\
\hline & $N(\%)$ & $N(\%)$ & $N(\%)$ & \\
\hline High-risk types (HR) & & & & 0.277 \\
\hline 16 & $3(21.4)$ & $11(78.6)$ & $14(6.1)$ & \\
\hline 18 & $6(46.2)$ & $7(53.9)$ & $13(5.7)$ & \\
\hline 26 & $1(50.0)$ & $1(50.0)$ & $2(0.9)$ & \\
\hline 31 & $6(46.2)$ & $7(53.9)$ & $13(5.7)$ & \\
\hline 33 & $7(50.0)$ & $7(50.0)$ & $14(6.1)$ & \\
\hline 35 & $5(33.3)$ & $10(66.7)$ & $15(6.6)$ & \\
\hline 39 & $1(50.0)$ & $1(50.0)$ & $2(0.9)$ & \\
\hline 45 & $3(27.3)$ & $8(72.7)$ & $11(4.8)$ & \\
\hline 51 & $4(44.4)$ & $5(55.6)$ & $9(4.0)$ & \\
\hline 52 & $4(22.2)$ & $14(77.8)$ & $18(7.9)$ & \\
\hline 53 & $5(20.8)$ & $19(79.2)$ & $24(10.5)$ & \\
\hline 56 & $5(38.5)$ & $8(61.5)$ & $13(5.7)$ & \\
\hline 58 & $4(57.1)$ & $3(42.9)$ & $7(3.1)$ & \\
\hline 59 & $5(50.0)$ & $5(50.0)$ & $10(4.4)$ & \\
\hline 66 & $2(16.7)$ & $10(83.3)$ & $12(5.3)$ & \\
\hline 68 & $8(38.1)$ & $13(61.9)$ & $21(9.2)$ & \\
\hline 69 & 0 & $2(100.0)$ & $2(0.9)$ & \\
\hline 73 & $2(10.5)$ & $17(89.5)$ & $19(8.3)$ & \\
\hline 82 & $1(11.1)$ & $8(88.9)$ & $9(4.0)$ & \\
\hline All & $72(31.6)$ & $156(68.4)$ & $228(100.0)$ & \\
\hline Low-risk types (LR) & & & & 0.202 \\
\hline 6 & $6(66.7)$ & $3(33.3)$ & $9(6.2)$ & \\
\hline 11 & 0 & $2(100.0)$ & $2(1.4)$ & \\
\hline 40 & $2(13.3)$ & $13(86.7)$ & $15(10.3)$ & \\
\hline 42 & $10(40.0)$ & $15(60.0)$ & $25(17.2)$ & \\
\hline 43 & $4(30.8)$ & $9(69.2)$ & $13(9.0)$ & \\
\hline 44 & $7(35.0)$ & $13(65.0)$ & $20(13.8)$ & \\
\hline 54 & $6(21.4)$ & $22(78.6)$ & $28(19.3)$ & \\
\hline 61 & $2(25.0)$ & $6(75.0)$ & $8(5.5)$ & \\
\hline 70 & $9(36.0)$ & $16(64.0)$ & $25(17.2)$ & \\
\hline All & $46(31.7)$ & $99(68.3)$ & $145(100.0)$ & \\
\hline TOTAL (HR and LR) & $118(31.6)$ & $255(68.4)$ & $373(100)$ & \\
\hline
\end{tabular}

${ }^{*} p$ value Mono-infections vs. Multi-infections

\section{Conclusions}

In conclusion, our study provides evidence that selfHPV with dry swabs stored at room temperature is a valid alternative for screening with HPV testing in low resource settings or remote areas, though HPV analysis should be performed ideally within 2 weeks. Moreover, our findings suggest that unsupervised self-sampling with dry swabs in conjunction with a PCR-based method is feasible in conducting population-based surveys on
HPV prevalence, which are limited or missing in many LMIC.

\section{Ethical approval}

Ethical approval of the study was obtained from the Malgasch National Commission for the Ethics of Science and Technology, as well as from the Ethical Cantonal Board of Geneva, Switzerland (CCER, CER: 14-071). 


\section{Abbreviations}

CC: cervical cancer; HPV: human papillomavirus; hrHPV: high-risk HPV; LMIC: low- and medium-income countries; IrHPV: low-risk HPV; self-HPV: self-sampling for HPV testing.

\section{Competing interests}

The authors declare that they have no competing interests.

\section{Authors' contributions}

Conceived and designed the experiments: PV RC UMH PP. Performed the experiments: SB MM CB UMH JJ JLH. Analysed the data: RC PV. Contributed reagents/materials/analysis tools: SB MM JJ JLH. Wrote the paper: PV RC SB PP. All authors read and approved the final manuscript.

\section{Funding}

This study was supported by the Action Madagascar Foundation and the University Hospitals of Geneva, Switzerland. The funders had no role in study design, data collection and analysis, decision to publish, or preparation of the manuscript.

\section{Author details}

'Geneva Foundation for Medical Education and Research, Route de Ferney 150, 1211 Geneva, Switzerland. Division of Gynaecology, Department of Gynaecology and Obstetrics, Geneva University Hospitals, Boulevard de la Cluse 30, 1206 Geneva, Switzerland. 'Biopath Lab SA, Rue du Liseron 11,

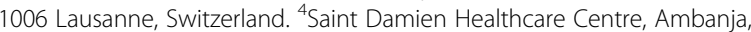
Madagascar.

\section{Received: 23 December 2015 Accepted: 15 February 2016} Published online: 17 March 2016

\section{References}

1. Schiffman M, Castle PE, Jeronimo J, Rodriguez AC, Wacholder S. Human papillomavirus and cervical cancer. Lancet. 2007;370(9590):890-907.

2. Vassilakos $P$, Catarino R, Frey TB, Petignat P. Cervical cancer screening in Switzerland: time to rethink the guidelines. Swiss Med Wkly. 2015;145: w14112.

3. World Health Organization. WHO guidelines for screening and treatment of precancerous lesions for cervical cancer prevention: supplemental material: GRADE evidence-to-recommendation tables and evidence profiles for each recommendation. 2013.

4. Arrossi S, Thouyaret L, Herrero R, Campanera A, Magdaleno A, Cuberli M, et al. Effect of self-collection of HPV DNA offered by community health workers at home visits on uptake of screening for cervical cancer (the EMA study): a population-based cluster-randomised trial. Lancet Glob Health. 2015;3(2):e85-94

5. Gravitt PE, Belinson JL, Salmeron J, Shah KV. Looking ahead: a case for human papillomavirus testing of self-sampled vaginal specimens as a cervical cancer screening strategy. Int J Cancer. 2011;129(3):517-27.

6. Crofts V, Flahault E, Tebeu P-M, Untiet S, Fosso GK, Boulvain M, et al. Education efforts may contribute to wider acceptance of human papillomavirus self-sampling. Int J Womens Health. 2015;7:149.

7. Catarino Jr R, Vassilakos P, Stadali-Ullrich H, Royannez-Drevard I, Guillot C, Petignat P. Feasibility of at-home self-sampling for HPV testing as an appropriate screening strategy for nonparticipants in Switzerland: preliminary results of the DEPIST study. J Low Genit Tract Dis. 2015;19(1):27-34.

8. Untiet S, Vassilakos P, McCarey C, Tebeu PM, Kengne-Fosso G, Menoud PA et al. HPV self-sampling as primary screening test in sub-Saharan Africa: implication for a triaging strategy. Int J Cancer. 2014;135(8):1911-7.

9. Ogilvie G, Patrick D, Schulzer M, Sellors J, Petric M, Chambers K, et al. Diagnostic accuracy of self collected vaginal specimens for human papillomavirus compared to clinician collected human papillomavirus specimens: a meta-analysis. Sex Transm Infect. 2005;81(3):207-12.

10. Petignat $P$, Faltin DL, Bruchim I, Tramèr MR, Franco EL, Coutlée F. Are selfcollected samples comparable to physician-collected cervical specimens for human papillomavirus DNA testing? A systematic review and meta-analysis. Gynecol Oncol. 2007;105(2):530-5.

11. Arbyn M, Verdoodt F, Snijders PJ, Verhoef VM, Suonio E, Dillner L, et al. Accuracy of human papillomavirus testing on self-collected versus cliniciancollected samples: a meta-analysis. Lancet Oncol. 2014;15(2):172-83.
12. Sultana F, Gertig DM, Wrede CD, English DR, Simpson JA, Drennan KT, et al. A pilot study to compare dry cervical sample collection with standard practice of wet cervical samples for human papillomavirus testing. J Clin Virol. 2015:69:210-3.

13. Wolfrum SG, Koutsky LA, Hughes JP, Feng Q, Xi LF, Shen Z, et al. Evaluation of dry and wet transport of at-home self-collected vaginal swabs for human papillomavirus testing. J Med Microbiol. 2012;61(Pt 11):1538-45.

14. Eperon I, Vassilakos P, Navarria I, Menoud P-A, Gauthier A, Pache J-C, et al. Randomized comparison of vaginal self-sampling by standard vs. dry swabs for human papillomavirus testing. BMC Cancer. 2013;13(1):353.

15. Haguenoer K, Giraudeau B, Gaudy-Graffin C, de Pinieux I, Dubois F, TrignolViguier $N$, et al. Accuracy of dry vaginal self-sampling for detecting high-risk human papillomavirus infection in cervical cancer screening: a crosssectional study. Gynecol Oncol. 2014;134(2):302-8.

16. Arundhati R, Young S, Erlich $H$, Boyle S, Krevolin M, Sun R, et al. Development and characterization of the cobas human papillomavirus test. J Clin Microbiol. 2013;51(12):4288.

17. Estrade C, Sahli R. Comparison of Seegene Anyplex II HPV28 with the PGMY-CHUV assay for human papillomavirus genotyping. J Clin Microbiol. 2014;52(2):607-12

18. Kim Y-J, Kwon M-J, Woo H-Y, Paik S-Y. Prevalence of human papillomavirus infection and genotype distribution determined by the cyclic-catcher melting temperature analysis in Korean medical checkup population. J Microbiol. 2013;51(5):665-70.

19. Kwon M-J, Roh KH, Park H, Woo H-Y. Comparison of the Anyplex II HPV28 assay with the Hybrid Capture 2 assay for the detection of HPV infection. J Clin Virol. 2014;59(4):246-9.

20. Bruni L, Barrionuevo-Rosas L, Albero G, Aldea M, Serrano B, Valencia S, Brotons M, Mena M, Cosano R, Muñoz J, Bosch FX, de Sanjosé S, Castellsagué $X$. ICO Information Centre on HPV and Cancer (HPV Information Centre). Human Papillomavirus and Related Diseases in Madagascar. Summary Report 2015-03-20. [Last accessed in 15 August 2015].

21. Feng $\mathrm{Q}$, Cherne $\mathrm{S}$, Winer RL, Popov V, Zambrano H, Yerovi C, et al. Evaluation of transported dry and wet cervical exfoliated samples for detection of human papillomavirus infection. J Clin Microbiol. 2010:48(9):3068-72.

22. Baay M, Verhoeven V, Lambrechts H, Pattyn G, Lardon F, Van Royen P, et al. Feasibility of collecting self-sampled vaginal swabs by mail: quantity and quality of genomic DNA. Eur J Clin Microbiol Infect Dis. 2009;28(11):1285-9.

23. Lin C, Cui J, Chen W, Qiao Y. Evaluation of the stability of cervical specimens collected by swab and stored dry for human papillomavirus DNA testing. Ann Glob Health. 2015;81(1):208.

24. Bessetti J. An introduction to PCR inhibitors. J Microbiol Methods. 2007;28: 159-67.

25. Bruni L, Diaz M, Castellsagué M, Ferrer E, Bosch FX, de Sanjosé S. Cervical human papillomavirus prevalence in 5 continents: meta-analysis of 1 million women with normal cytological findings. J Infect Dis. 2010;202(12):1789-99.

26. Trottier H, Ferreira S, Thomann P, Costa MC, Sobrinho JS, Prado JCM, et al. Human papillomavirus infection and reinfection in adult women: the role of sexual activity and natural immunity. Cancer Res. 2010;70(21):8569-77.

27. Ogembo RK, Gona PN, Seymour AJ, Park HS-M, Bain PA, Maranda L, et al. Prevalence of human papillomavirus genotypes among African women with normal cervical cytology and neoplasia: a systematic review and meta-analysis. 2015

28. Castellsagué X, Menéndez C, Loscertales M-P, Kornegay JR, dos Santos F, Gómez-Olivé FX, et al. Human papillomavirus genotypes in rural Mozambique. Lancet. 2001;358(9291):1429-30

29. Bosch FX, Burchell AN, Schiffman M, Giuliano AR, de Sanjose S, Bruni L, et al. Epidemiology and natural history of human papillomavirus infections and type-specific implications in cervical neoplasia. Vaccine. 2008;26:K1-16.

30. Krech T, Castriciano S, Jang D, Smieja M, Enders G, Chernesky M. Detection of high risk HPV and Chlamydia trachomatis in vaginal and cervical samples collected with flocked nylon and wrapped rayon dual swabs transported in dry tubes. J Virol Methods. 2009;162(1):291-3.

31. Li J, Jang D, Gilchrist J, Smieja M, Ewert R, MacRitchie C, et al. Comparison of flocked and Aptima swabs and two specimen transport media in the Aptima Combo 2 assay. J Clin Microbiol. 2014;52(10):3808-9.

32. Brownlow RJ, Dagnall KE, Ames CE. A comparison of DNA collection and retrieval from two swab types (cotton and nylon flocked swab) when processed using three QIAGEN extraction methods. J Forensic Sci. 2012; 57(3):713-7. 